\title{
Antibacterial activity of the rose extract
}

\author{
Yusra Safdar and Taqdees Malik* \\ Department of Microbiology, Jinnah University for Women, Pakistan
}

*Corresponding author: Taqdees Malik, Department of Microbiology, Jinnah University for Women, Karachi, Pakistan

\begin{abstract}
Antimicrobial agents are defined as those substances which possess inhibitory effects against gram-positive and gram-negative bacteria, preventing their growth. Antibacterial agents are classified based on the type of action, source of antibacterial agent, and range of the spectrum. Rose belongs to the family Rosaceae. Hundred $(n=100)$ species of roses are locally available. Roses are native primarily to the temperate regions of the Northern Hemisphere. There is a wide variety of colors available in roses which enhances the beauty of the rose. Most roses are locally available in Asia, North America and few are available in Europe. Many parts of rose have been used to check the antibacterial and antifungal property and they are highly active to remove toxins from the body and shown positive results to inhibit the growth of bacteria. Different parts of rose can be used separately against bacteria. Rose extract can also be used to treat sore throat and to relieve chest congestion. The agents which are responsible for the antibacterial and antifungal properties of the rose extract are flavonoid and phenolic compounds. The rose petals are powdered and mixed in distilled water and concentrated using a rotary evaporator. Pure cultures were swabbed on MHA plates and wells were made using sterile borer by agar well diffusion method. Twenty (20ul) of the extract was added in well and incubated at $37^{\circ} \mathrm{C}$ for 24 hours. Next day zone of inhibition was observed. To compare the activity of rose extract with amikacin, pure cultures were swabbed on MHA and amikacin disk was placed on each plate. Next day zones were observed. Rose extract has shown maximum inhibitory effects against Staphylococcus aureus whereas the inhibitory effects against Bacillus cereus, Bacillus subtilis, and Klebsiella pneumoniae were not satisfactory. We have also observed that Vibrio cholera also showed sensitivity to rose extract whereas E.coli and Pseudomonas aureginosa gave intermediate zones, therefore we suggest that we can use rose extract against Staph aureus, E.coli, Pseudomonas aureginosa and Vibrio cholera.
\end{abstract}

Keywords: Rose extract; Antibacterial activity; Sensitivity; Resistance; Disc diffusion

\section{Introduction}

Rose belongs to the family Rosaceae. Hundred ( $\mathrm{n}=100)$ species of roses are locally available. Roses are native primarily to the temperate regions of the Northern Hemisphere. There is a wide variety of colors available in roses which enhances the beauty of the rose. Most roses are locally available in Asia, North America and few are available in Europe. There are so many important components of rose which plays an important role in the antibacterial activity of rose. Among them, the components which possess antibacterial activity are flavonoids, terpenes, anthocyanins. Flavonoids are classified as natural plant compounds usually they are secondary metabolites of plants with various phenolic structures saul riuz cruz et al. [1]. They have many properties that help to treat cancer Alzheimer's disease like they pose biochemical and antioxidant effects, antimutagenic effect, anticarcinogenic effects, etc. A.N Panche et al. [2]. They are extracted from plants and are classified as a low molecular weight compound. Rose, onion is the major source of flavonoids Aleksandra et al. [3]. Apart from antibacterial and antifungal properties, flavonoids are also responsible for color and aroma production in flowers Dr. Nicola. [4]. They are helpful for plants in a way that they act as a UV filter which protects the DNA of plant from UV radiations Burak $\mathrm{M}$ et al. [5]. Classification is done based on $\mathrm{C}$ ring which contains carbon Calhoun et al. [6]. Terpene is a biological compound present in the rose flower. Terpenes have great industrial and pharmaceutical usage. In food industries, it is used as a flavoring agent or fragrancing agent. Pharmaceutical industries have medicinal uses of terpene Jiang $\mathrm{z}$ et al. [7]. Concentrates in ongoing decades have shown that terpenes apply anti-inflammatory impacts by hindering different proinflammatory pathways. Terpenes have been shown to exert activity against cancer and tumors Cho KS et al. [8].

Anthocyanins belong to flavonoids class which includes a subset of the polyphenol. Red, blue, and purple shades of organic 
products, vegetables, grains, blossoms, and herbs are due to anthocyanin. Anthocyanin is a Greek word, anthos means to bloom and kyanos means blue. Anthocyanins are transcendently found in nature as glycosides of polyhydroxy and polymethoxy subordinates of 2-phenyl-benzopyryliurn or flavylium salts. They are separated by the quantity of hydroxyl and methoxyl types of the B-ring, by the number of sugars connected to the aglycon and the situation of connection, and by the nature and number of aliphatic or aromatic acids appended to the sugar deposits welch et al. [9]. In a past report, quiniccorrosiv, 5-hydroxymethylfurfural, pyrogallol, levoglucosan, and 4H-pyran-4- one, 2, 3-dihydro-3, 5-dihydroxy-6-methyl were the major distinguished segments in methanolic concentrate of $\mathrm{R}$. indica petals. In like manner, another examination demonstrated that unstable oils of new blooms of R. Damascena, for the most part, have citronellol, geraniol, nonadecane, and heneicosane in the fundamental oil, while they have alcoholic segments, citronellol and geraniol in rose water portion bai S. et al. [10].

Several studies have been done on rose and its products. It was found that several diseases can be treated with rose products and rose extracts. A recent study was done to check the antiviral activity of rose flower. In this research rose petals extract were made with water and ethanol separately. Now, this extract used against HIV. Rose petals extract showed antiviral activity against HIV. It inhibits the activity of HIV by interfering in replication at different stages. A common flavonoid known as kaempferol found in rose petals inhibits the activity of viral proteases Pon velayutham et al. [11] Mahmood n, et al. [12]. Rose extract also used against several bacterial species to treat various bacterial infections. It is effective against both gram-positive and gram-negative bacteria. But on the other hand, several rose species possess no antibacterial activity, it includes Bulgarian rose Kalemba d et al. [13]. When the rose extract was made with different solutions it shows that rose extract along with petroleum ether showed great antibacterial activity as compared to rose extract with water or ethanol hirulkar n, b [14]. Without a doubt, the antimicrobial action of rose concentrates particularly rose fundamental oil is identified with compound segments particularly geraniol, citronellol and nerol or interdependent impacts between these segments. The antibacterial and antifungal exercises of geraniol were affirmed against an enormous number of microorganisms. Likewise, the interdependent impact between citronellol, geraniol and nerol were shown against Gram-positive, Gram-negative bacteria. On the other hand, the antimicrobial movement of rose concentrates is identified with compound parts of concentrates and their interdependent or adversarial impacts andogan B.C et al. [15]. Several compositions of the rose extract showed anti-cancerous activity. The geraniol as the fundamental mixes of R. Damascena's acts using various systems. It causes the apoptosis in malignant growth cells and expands the outflow of apoptotic protein Bak47 captures the G0/G1 period of cell cycle and diminishes cdk2 activity, restrains the 3-hydroxy-3-methylglutaryl-CoA (HMG-CoA) reductase and ornithine decarboxylase activity that at last causes the passing in diseased cells Elson C, E [16]. Rose extract also showed antidepressant activity. R. Damascena using animating the $\beta$-adrenergic receptors repressing the histamine $\mathrm{H} 1$ receptors and obstructing the calcium channels of the tracheal chain, hindering the $\mathrm{KCl}$-related withdrawal and electrical field incitement thus acts as a relaxant boskabady $\mathrm{m}$. et al. [17]. The antidepressant impacts of $R$. Damascena fluid concentrate were affirmed in animal models. Rose total shows the energizer movement by diminishing the lipid peroxidation and expanding the cancer prevention agents in the cerebral cortex. As referenced previously, it is accepted that rose fundamental oil and rose water bring joy, fearlessness and are known as an erotic and sexual enhancer. It has been affirmed that rose fundamental oil used in infertility and drive through expanding the widths of seminiferous tubules, sperm check, and motility and upgrading the testosterone production. Furthermore, the organization of rose basic oil improves sexual brokenness and manifestations of depression in male patients experiencing significant depression issues Farina v et al. [18]. Rose extract also showed antioxidant activity. Phenolic compounds and flavonoids are responsible for the antioxidant activity of rose. The advantages impact of rose fundamental oil against formaldehyde inward breath on the reproductive system are identified with the cancer prevention agent movement of rose basic oil.

\section{Material and Methods \\ Sample collection}

The rose petals were collected from the local flower market. The petals were dried under shade for 1 week, pulverized into fine particles Devyani Bahl et al. [19].

\section{Preparation of rose extract}

The petals were dried under shade for 1 week, pulverized into fine particles by using a home chopper machine. The extract was prepared using distilled water. $200 \mathrm{~g}$ of fine powder of rose petal was mixed in distilled water. Then it was concentrated using a rotary evaporator. The mixture was strained using Whatman filter paper and was placed on the funnel to obtain a filtrate. The filtrate was refrigerated for further use.

\section{Identification of Cultures and Biochemical Testing}

Biochemical tests are the tests utilized for the recognizable proof of microscopic organism's species dependent on the distinctions in the biochemical characteristics of various microorganisms. The biochemical test used for gram-positive strains is catalase, oxidase, coagulase, and urease. Catalase test is utilized to distinguish bacteria that produce the catalase. This catalyst detoxifies hydrogen peroxide by separating it into water and oxygen gas. Coagulase test is used to check the ability of an organism to clot blood plasma. Oxidase test is used to check the presence of an enzyme cytochrome 
oxidase that transfers electron in the electron transport chain. A urease test is done to identify that an organism can utilize urea or not. As a result of the breakdown of urea, ammonia is produced. For gram-negative organisms, the IMVIC test is used. IMVIC test contains an indole test, methyl red test, Voges Proskauer test and citrate test. Along with IMVIC, the TSI test is also performed. TSI is a triple sugar iron test in which an organism utilizes sugar and produced hydrogen sulfide

\section{Antibacterial activity/susceptibility testing}

To assess the antibacterial activity of the rose extract, eight cultures were collected from the known clinical laboratory of Karachi. Gram-positive cultures include S. aureus, B. cereus, B. subtilis. Gram-negative cultures include Pseudomonas aeruginosa, Vibrio cholera, E. coli, Klebsiella pneumonia, Enterobacter. They were all gram stained and confirmed by biochemical testing and then they were cultured on nutrient agar plates and preserved for further use.

The antibacterial activity of the rose extract was analyzed against these isolates by using Tryptic Soy Agar (TSA) plates, by

Table 1: Morphological and cultural characteristics of identified strains.

\begin{tabular}{|c|c|c|}
\hline Organisms & Gram Reaction & Cultural Characteristics \\
\hline Staphylococcus aureus & Gram-positive & Large, circular, smooth, shiny, and pigmented on nutrient agar. \\
\hline Bacillus cereus & Gram-positive & Grey white irregular colonies on nutrient agar. Beta hemolysis on blood agar \\
\hline Bacillus subtilis & Gram-positive & Rough colonies on nutrient agar. Beta hemolysis on blood agar. \\
\hline E. coli & Gram-negative & Regular, flat whitish translucent colonies on nutrient agar. On EMB E. coli gives a green metallic sheen \\
\hline Pseudomonas aeroginosa & Gram-negative & $\begin{array}{l}\text { Regular, slightly greenish, and translucent colonies on nutrient agar. It gives colorless colonies on EMB. } \\
\text { On Psuedo agar, it gives green pigmentation. }\end{array}$ \\
\hline Klebsiella pneumonia & Gram-negative & Lactose fermenting, mucoid pink colonies on MacConkey agar. \\
\hline Vibrio cholera & Gram-negative & $\begin{array}{c}\text { Glistening translucent colonies on nutrient agar. On MacConkey it gives non lactose fermenting } \\
\text { colonies. }\end{array}$ \\
\hline Enterobacter & Gram-negative & Small grey colonies blood agar. Nutrient agars have colorless colonies \\
\hline
\end{tabular}

Table 2: Biochemical characteristics of gram-positive strains.

\begin{tabular}{|c|c|c|c|c|}
\hline \multirow{2}{*}{ Organisms } & \multicolumn{3}{|c|}{ Gram Positive Biochemical Test } \\
\cline { 2 - 5 } & Catalase & \multicolumn{2}{|c|}{ Oxidase } & \multicolumn{2}{|c|}{ Urease } & + \\
\hline S. aureus & + & - & - \\
\hline B. cereus & + & - & - \\
\hline B. subtilis & + & - & - \\
\hline
\end{tabular}

Table 3: Biochemical characteristics of gram-negative strains.

\begin{tabular}{|c|c|c|c|c|}
\hline \multicolumn{2}{|c|}{ Gram Negative Biochemical Test } \\
\hline \multirow{2}{*}{ Organisms } & \multicolumn{3}{|c|}{ Biochemical Test } \\
\cline { 2 - 5 } & Indole & Methyl Red & VP & Citrate \\
\hline E. coli & + & + & - & + \\
\hline Psedumomans & - & - & + & + \\
\hline Klebsiella & - & - & + & + \\
\hline Vibrio & - & - & AK-- & + \\
\hline Enterobacter & - & KA-- & + \\
\hline
\end{tabular}

Citation: Yusra Safdar, Taqdees Malik. Antibacterial activity of the rose extract. Open Acc J Comp \& Alt Med 2(4) - 2020. OAJCAM. 


\section{Antibacterial activity assay}

The antibacterial activity is checked against all seven test organisms. The results of the zone of inhibition showed in Table 4 and Figure 1-3. Table 5 and Figure 4-8.

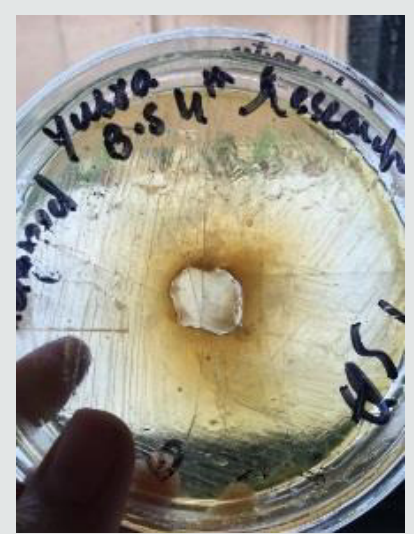

Figure 1: Staph. aureus

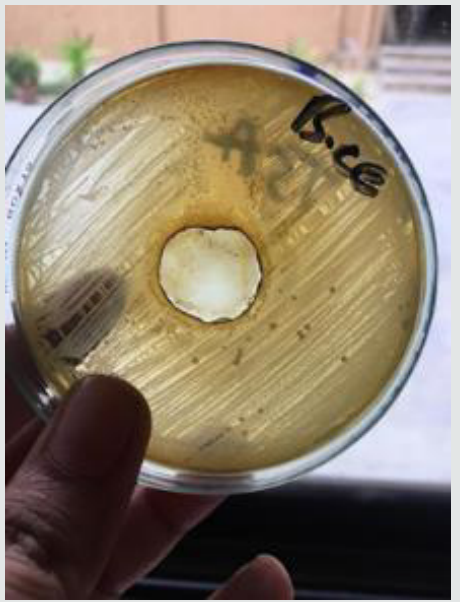

Figure 2: B. cereus

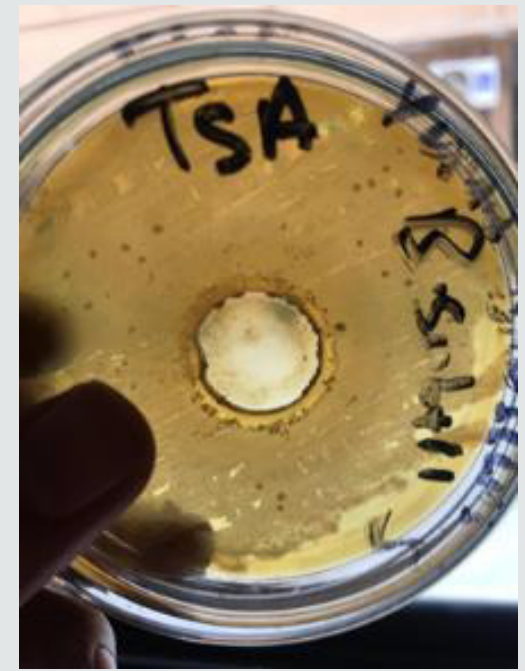

Figure 3: B. subtilis

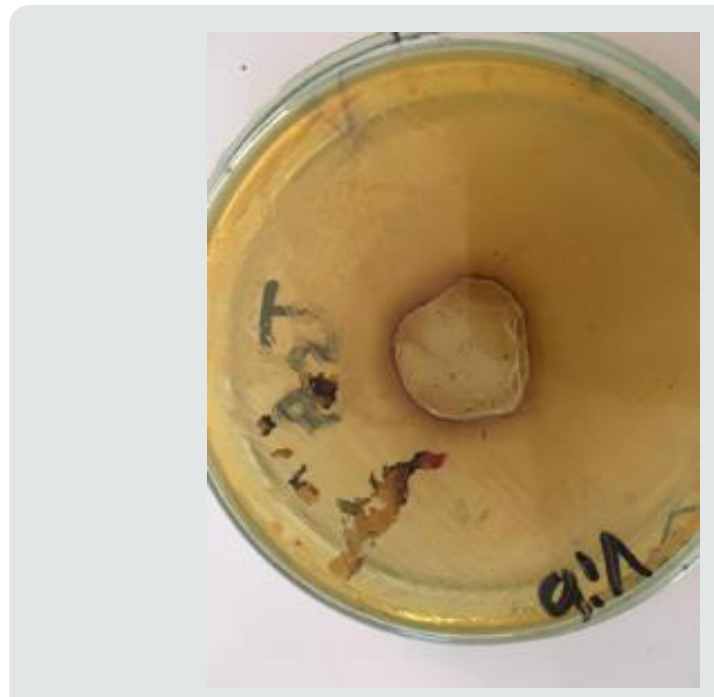

Figure 4: Vibrio cholera

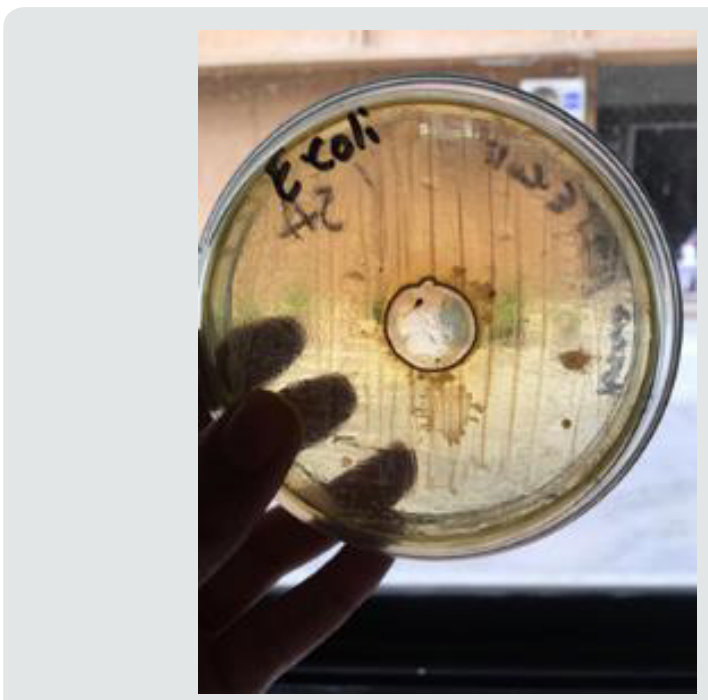

Figure 5: E.coli

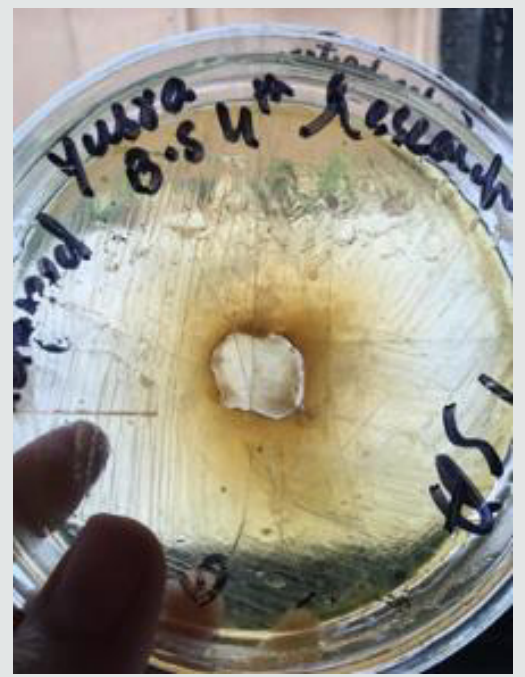

Figure 6: $P$ aureginosa 


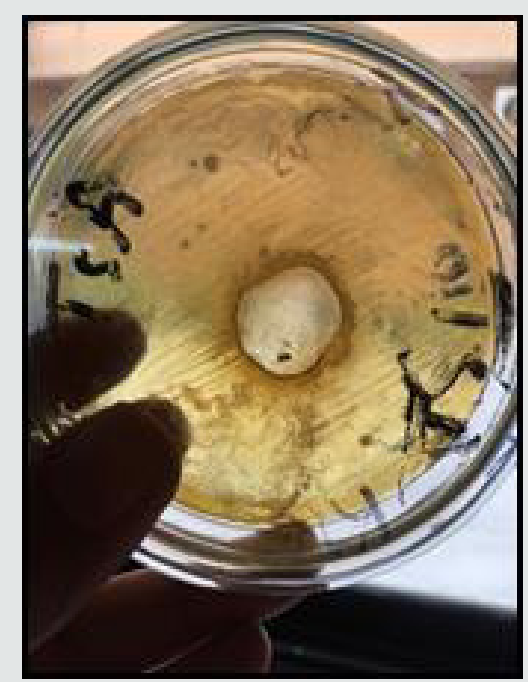

Figure 7: K. pneumonia

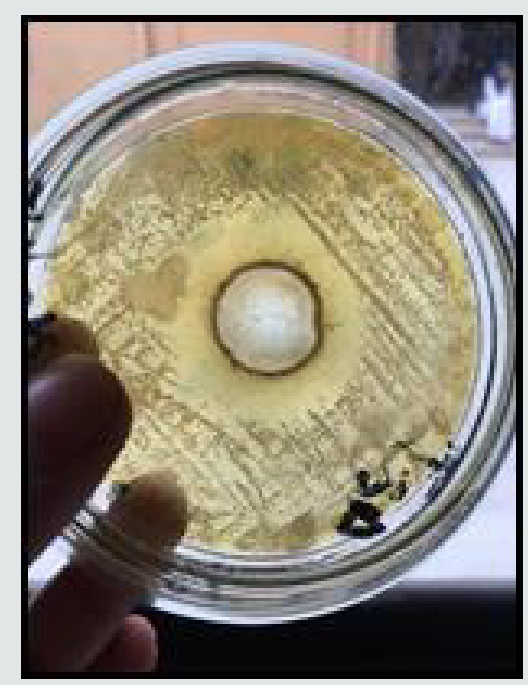

Figure 8: Enterobacter

Table 4:

\begin{tabular}{|c|c|}
\hline \multicolumn{2}{|c|}{ Susceptibility Testing of Rose Extract against Gram Positive } \\
\hline Organisms & Zone of Inhibition \\
\hline S. aureus & $35 \mathrm{~mm}$ \\
\hline B. cereus & $20 \mathrm{~mm}$ \\
\hline B. subtilis & $20 \mathrm{~mm}$ \\
\hline
\end{tabular}

Table 5:

\begin{tabular}{|c|c|}
\hline \multicolumn{2}{|c|}{ Susceptibility Testing of Rose Extract against Gram Negative } \\
\hline Organisms & Zone of Inhibition \\
\hline E. coli & $24 \mathrm{~mm}$ \\
\hline Pseudomonas aureginosa & $23 \mathrm{~mm}$ \\
\hline Klebsiella pneumonia & $20 \mathrm{~mm}$ \\
\hline Vibrio cholera & $29 \mathrm{~mm}$ \\
\hline Enterobacter & $44 \mathrm{~mm}$ \\
\hline
\end{tabular}

\section{Antibiotic susceptibility testing}

Table 6 and Figure 9-11.

Table 7 and Figure 12-16.

Table 6:

\begin{tabular}{|c|c|}
\hline \multicolumn{2}{|c|}{ Results of Gram-Positive Bacteria Against Amikacin } \\
\hline Organism & Zone of Inhibition \\
\hline S. aureus & $22 \mathrm{~mm}$ \\
\hline B. cereus & $23 \mathrm{~mm}$ \\
\hline B. subtilis & $24 \mathrm{~mm}$ \\
\hline
\end{tabular}

Table 7:

\begin{tabular}{|c|c|}
\hline \multicolumn{2}{|c|}{ Results of Gram-Negative Bacteria Against Amikacin } \\
\hline Organism & Zone of Inhibition \\
\hline E. Coli & $15 \mathrm{~mm}$ \\
\hline Pseudomonas & $21 \mathrm{~mm}$ \\
\hline Klebsiella & $19 \mathrm{~mm}$ \\
\hline Vibrio & $17 \mathrm{~mm}$ \\
\hline Enterobacter & $20 \mathrm{~mm}$ \\
\hline
\end{tabular}

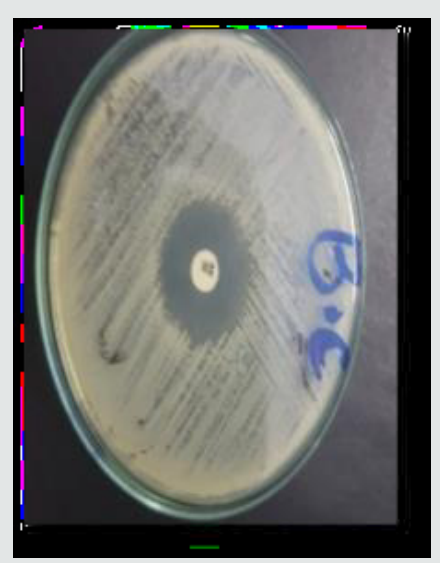

Figure 9: Bacillus cereus

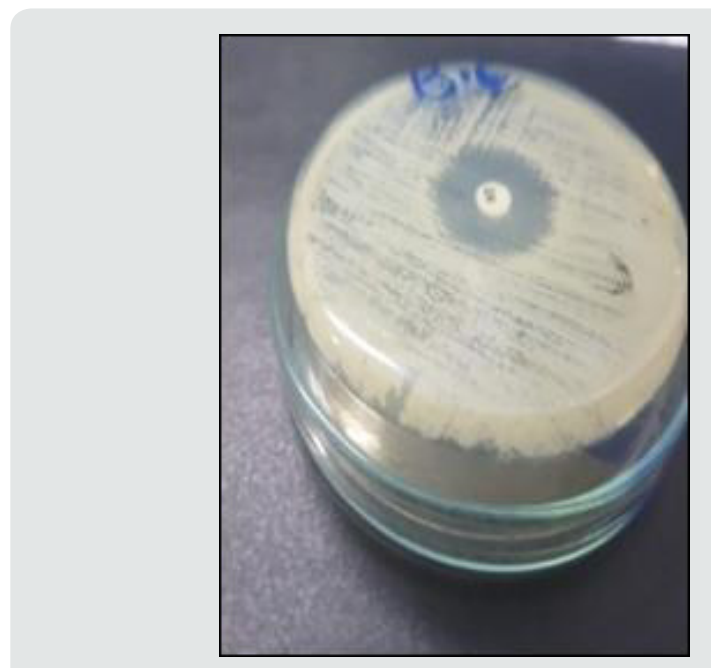

Figure 10: Bacillus subtilis 


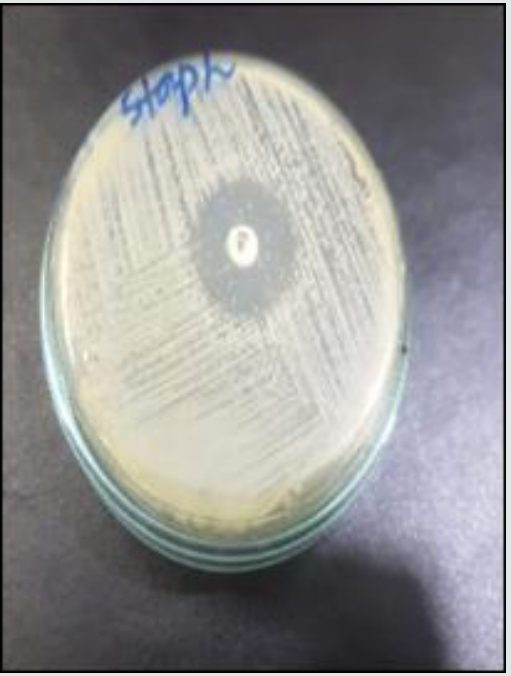

Figure 11: Staph aureus

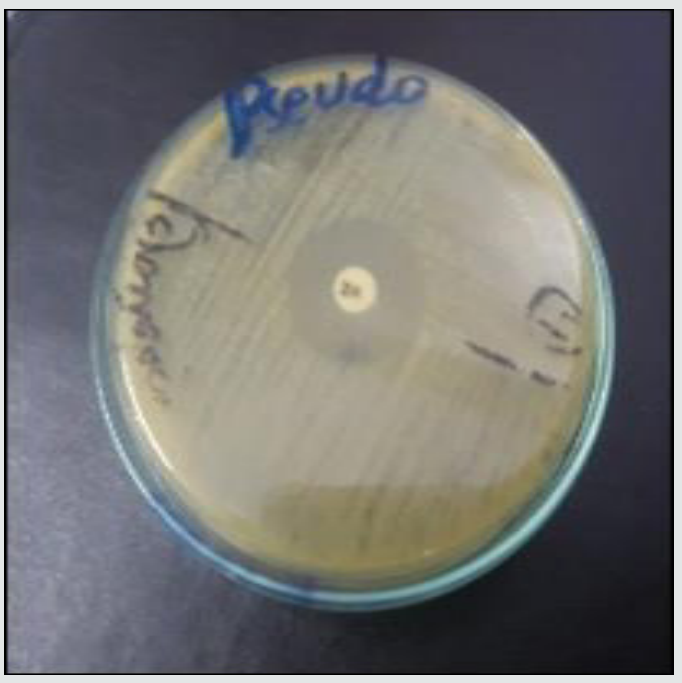

Figure 12: $P$. aeroginosa

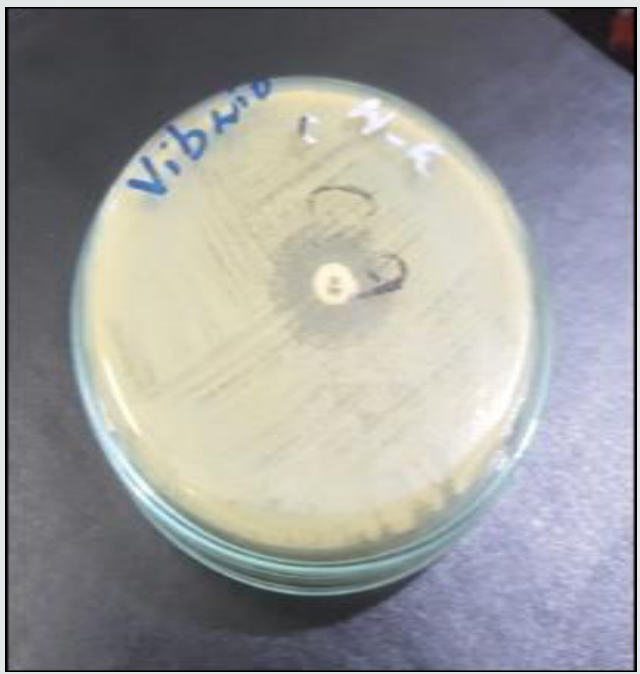

Figure 13: Vibrio cholera

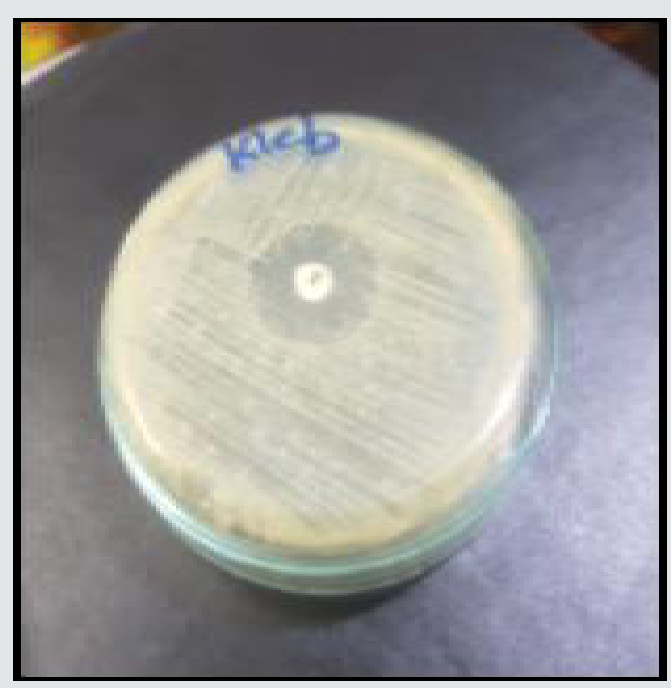

Figure 14: K.pneumoniae

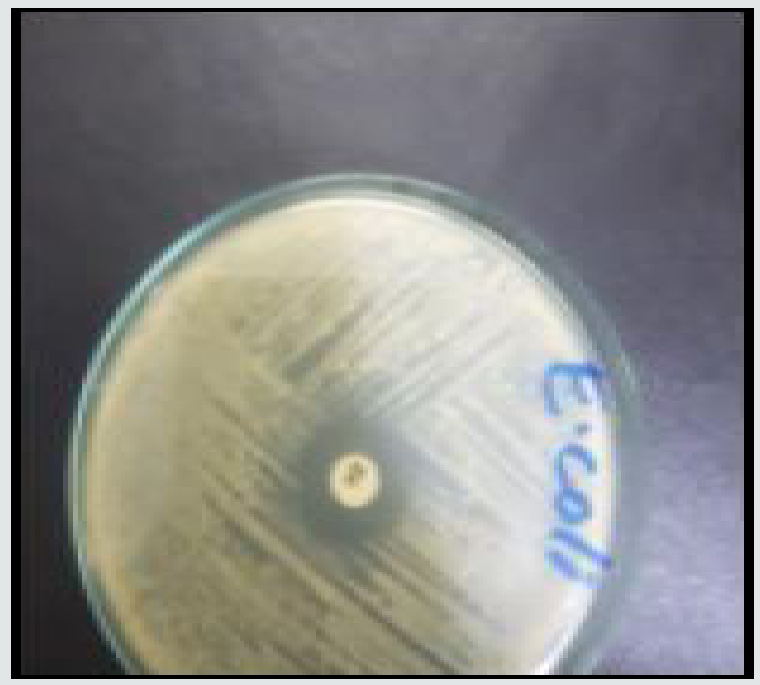

Figure 15: E.coli

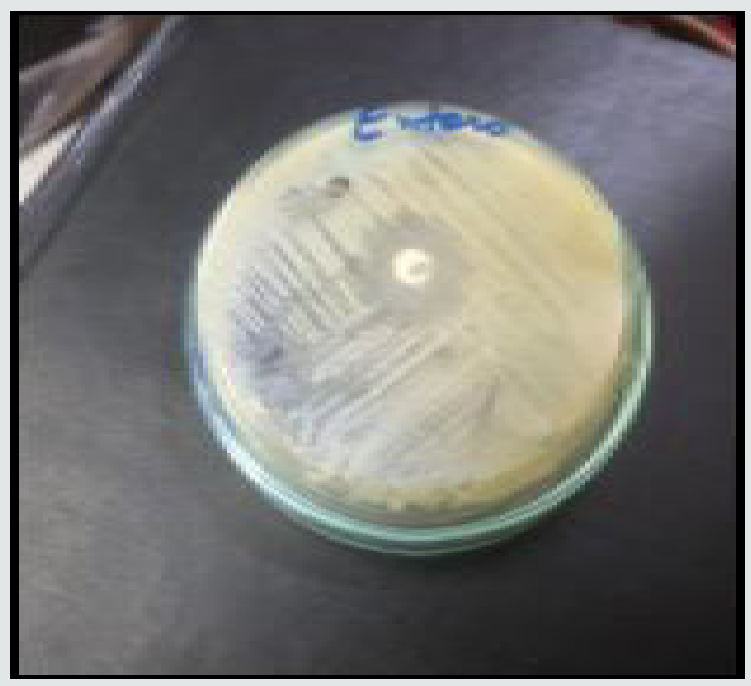

Figure 16: Enterobacter 


\section{Discussion}

Pathogenic microorganisms are the major cause of infectious illness worldwide because of their resistance ability. Today the most important need is the discovery of those natural agents that have the potential to kill or inhibit the growth of the microorganism and should have no toxic effects on the human body after consumption. Several types of research have been conducted to fulfill the requirement of the discovery of natural compounds. The traditional use of plants as medicines provide the basis for indicating which plant extract could be useful for specific medical conditions. In the past, many plant extracts such as rose extract, tea extract, clove extract have been used as an antibacterial agent which may help to treat several infections. It is important to investigate that the natural plants which possess antimicrobial activity can be used for large group or pathogens or not. Several studies have been conducted to check the antibacterial activity of various plants extract to prove that either they are helpful to treat various infections or not.

The present study represents the antibacterial activity of the rose extract. This plant contains a few parts, for example, terpenes, glycosides, flavonoids, and anthocyanins that affect the health of humans. The pharmacological impacts of $R$. damascene are vast. The majority of the CNS impacts are sleep-inducing, pain-relieving, and anticonvulsant impacts. The respiratory, cardiovascular, purgative, antidiabetic, antimicrobial, hostile to HIV, calming, and cancer prevention agent are different impacts of this plant. It is recommended that lipid dissolvable (non-polar) constituents of this plant are principally responsible for the vast majority of the previously mentioned impacts. According to results, the rose extract showed the highest activity against Enterobacter, S. aurus and vibrio cholera. But rose extract also showed activity against other tested microbes but to a lower extent.

To compare the activity of rose extract with an antibiotic, we selected amikacin to compare the antibacterial and antifungal activity. Amikacin is a novel antibiotic because it gives the best results against Pseudo and drug-resistant bacilli. It also showed activity against all tested strains.

\section{Conclusion}

This study shows there are many compounds present in rose flowers that possess antibacterial activity so it has confirmed that rose extract could be used for the treatment of various infections including multidrug-resistant staph aureus infections. Further studies should be done for antimicrobial screening of this product so that it can use in health care needs.

\section{Authors' Contributions}

Conceived and designed the experiments: T Malik, Performed the experiments: Y Safdar, Analyzed the data: T Malik \& Y Safdar, contributed reagents/ materials/ analysis tools: T Malik \& Y Safdar, Wrote the paper: Y Safdar.

\section{References}

1) Hamid Ullah and Saqibali. Classification of antibacterial agents and their functions. $31^{\text {st }}$ May 2017

2) GaurabKarki. Classification of antibiotics. February 3, 2018.

3) Calhoun C, Hall GA. Antibiotics. [Updated 2019 May 14]. In: StatPearls [Internet]. Treasure Island (FL): StatPearls Publishing; 2019 Jan

4) A. N Panche, A. D. Diwan, and S. R. Chandra. Flavonoids an overview. Journal of national science. 26 December 2016. pp 1-15

5) DevyaniBahl, Swati, Anirudh Chakravarthy, Srinivas Mutalik, RavirajDevkar. Determination of Antibacterial and Antifungal Properties of Rose Extract- an In vitro Study. International Journal of Pharmacognosy and Phytochemical Research 2016; 8(10); 1695-1697

6) DR. NICO LA MAZZINI. Flavonoid definition structure and classification. 22 January 2014

7) Aleksandra Kozłowska, Dorota Szostak-Węgierek2. FLAVONOIDS FOOD SOURCES AND HEALTH BENEFITS. Department of Preventive Medicine and Hygiene, Institute of Social Medicine, Medical University of Warsaw, Poland 2 Department of Human Nutrition, Faculty of Health Science, Medical University of Warsaw, Poland. RoczPanstwZaklHig 2014;65(2):79-85

8) Mohammad HosseinBoskabady, Mohammad NaserShafei, Zahra Saberi, SomayehAmini. Pharmacological Effects of Rosa Damascena. Iranian Journal of Basic Medical Sciences Vol. 14, No. 4, July-Aug 2011, 295-307

9) Saul Ruiz-Cruz, SaraíChaparro-Hernández, Karen L. Hernández- Ruiz, Luis A. Cira-Chávez, María I. Estrada-Alvarado, Laura E. Gassos Ortega, José de Jesús Ornelas-Paz and Marco A. Lopez Mata.Flavonoids: Important Biocompounds in Food. August 23rd, 2017

10) PonVelayuthamAnandh Babu, Dongmin Liu. Flavonoids and Cardiovascular Health. 2009

11) Kalemba D, Kunicka A. Antibacterial and antifungal properties of essential oils. US national library of medicines. Pubmed. 2003 May; 10(10):813-29.

12) Ramirez, M. S., \&Tolmasky, M. E. (2017). Amikacin: Uses, Resistance, and Prospects for Inhibition. Molecules (Basel, Switzerland), 22(12), 2267. DOI:10.3390/molecules22122267

13) Joods-Panzaru, S., Nelson, D., McCollum, G., Ballard, L. M., Millar, B. C., Maeda, Y., ... Moore, J. E. (2009). An examination of antibacterial and antifungal properties of constituents described in traditional Ulster cures and remedies. The Ulster medical journal, 78(1), 13-15.

14) Sizar O, Sundareshan V. Amikacin. [Updated 2019 Oct 12]. In: StatPearls [Internet]. Treasure Island (FL): StatPearls Publishing; 2019 Jan.

15) Burak M \&Imen $Y$ (1999) Flavonoids and their antioxidant properties. TurkiyeKlin Tip BilDerg 19, 296-304.

16) Dixon R \&Pasinetti G (2010) Flavonoids and isoflavonoids: from plant biology to agriculture and neuroscience. Plant Physiol 154, 453-457

17) Boskabady, M. H., Shafei, M. N., Saberi, Z., \&Amini, S. (2011). Pharmacological effects of Rosa Damascena. Iranian journal of basic medical sciences, 14(4), 295-307.

18) Mármol, I.,Sánchez-de-Diego, C.,Jiménez-Moreno, N.,Ancín-Azpilicueta, C., \& Rodríguez-Yoldi, M. J. (2017). Therapeutic Applications of Rose Hips from Different Rosa Species. International journal of molecular sciences, 18(6), 1137. DOI:10.3390/ijms18061137 
19) Mahboubi M. (2015). Rosa damascena as a holy ancient herb with novel applications. Journal of traditional and complementary medicine, 6(1), 10-16. DOI:10.1016/j.jtcme.2015.09.005

20) Mahmood N., Piacente S., Pizza C., Burke A., Khan A.I., Hay A.J. The antiHIV activity and mechanisms of action of pure compounds isolated from Rosa damascena. Biochim Biophys Res Commun. 1996; 229:7379.

21) Hirulkar N.B. Antimicrobial activity of rose petals extracts against some pathogenic bacteria. Int J Pharm Biol Arch. 2010; 1:478-484.

22) Andoğan B.C., Baydar H., Kaya S., Demirci M., Özbaşar D., Mumcu E. Antimicrobial activity and chemical composition of some essential oils. Arch Pharm Res. 2002; 25:860-864.

23) Elson C.E. Suppression of mevalonate pathway activities by dietary isoprenoids: protective roles in cancer and cardiovascular disease. J Nutr. 1995; 125(6 suppl):1666S-1672S.

24) Boskabady M., Kiani S., Rakhshandah H. Relaxant effects of Rosa damascena on guinea pig tracheal chains and its possible mechanism (s) J Ethnopharmacol. 2006;106:377-382

25) Farnia V., Shirzadifar M., Shakeri J. Rosa damascena oil improves SSRIinduced sexual dysfunction in male patients suffering from major depressive disorders: results from a double-blind, randomized, and placebo-controlled clinical trial. Neuropsychiatr Dis Treat. 2015; 11:625-635.

26) Halvorsen B.L., Holte K., Myhrstad M.C. A systematic screening of total antioxidants in dietary plants. J Nutr. 2002; 132:461-471.

27) Willich S., Rossnagel K., Roll S. Rose hip herbal remedy in patients with rheumatoid arthritis- a randomized controlled trial. Phytomedicine. 2010;17:87-93

28) Jiang, Z., Kempinski, C., \& Chappell, J. (2016). Extraction and Analysis of Terpenes/Terpenoids. Current protocols in plant biology, 1, 345-358. DOI:10.1002/cppb.20024

29) Cho, K. S., Lim, Y. R., Lee, K., Lee, J., Lee, J. H., \& Lee, I. S. (2017). Terpenes from Forests and Human Health. Toxicological Research, 33(2), 97106. DOI:10.5487/TR.2017.33.2.097

30) Welch, C. R., Wu, Q., \& Simon, J. E. (2008). Recent Advances in Anthocyanin Analysis and Characterization. Current analytical chemistry, 4(2), 75-101. DOI:10.2174/157341108784587795
31) Bai S., Seasotiya L., Malik A., Bharti P., Dalal S. Bioactive compounds and pharmacological potential of Rosa indica L. and Psidiumguajava L. methanol extracts as antiurease and anti-collagenase agents. Der Pharmacia Lettre. 2015; 7(1):179-184.

32) Boskabady MH, Vatanprast A, Parsee H, Ghasemzadeh M. Effect of aqueous-ethanolic extract from Rosa damascena on the guinea-pig isolated heart. Iran J Basic Med Sci. 2011; 14:116-121.

33) Gholamhoseinian A, Shahouzehi B, Sharififar F. Inhibitory effect of some plant extract on pancreatic lipase. Int J Pharmacol. 2010;6:18-24

34) Marrack J. R. (1953). BIOCHEMICAL TESTS. British Medical Journal, 2(4845), 1089.

35) Sarkar, P., Yarlagadda, V., Ghosh, C., \& Haldar, J. (2017). A review of cell wall synthesis inhibitors with an emphasis on glycopeptide antibiotics. MedChemComm, 8(3), 516-533. DOI:10.1039/c6md00585c

36) Shetty, A., \& Dick, T. (2018). Mycobacterial Cell Wall Synthesis Inhibitors Cause Lethal ATP Burst. Frontiers in microbiology, 9, 1898. DOI:10.3389/fmicb.2018.01898

37) Liu, Y., \& Breukink, E. (2016). The Membrane Steps of Bacterial Cell Wall Synthesis as Antibiotic Targets. Antibiotics (Basel, Switzerland), 5(3), 28. DOI:10.3390/antibiotics5030028

38) Sugimoto, A., Maeda, A., Itto, K., \& Arimoto, H. (2017). Deciphering the mode of action of cell wall-inhibiting antibiotics using metabolic labeling of growing peptidoglycan in Streptococcus pyogenes. Scientific reports, 7(1), 1129. DOI:10.1038/s41598-017-01267-5

39) Arenz, S., \& Wilson, D. N. (2016). Bacterial Protein Synthesis as a Target for Antibiotic Inhibition. Cold Spring Harbor perspectives in medicine, 6(9), a025361. DOI:10.1101/cshperspect.a025361

40) Franklin, T. J., \& Cook, J. M. (1969). The inhibition of nucleic acid synthesis by mycophenolic acid. The Biochemical journal, 113(3), 515524. DOI:10.1042/bj1130515

41) Bendahmane, M., Dubois, A., Raymond, O., \& Bris, M. L. (2013). Genetics and genomics of flower initiation and development in roses. Journal of experimental botany, 64(4), 847-857. DOI:10.1093/jxb/ers387

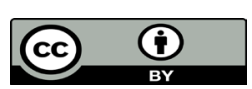

This work is licensed under Creative Commons Attribution 4.0 License

To Submit Your Article Click Here: Submit Article

DOI: 10.32474/OAJCAM.2020.02.000144

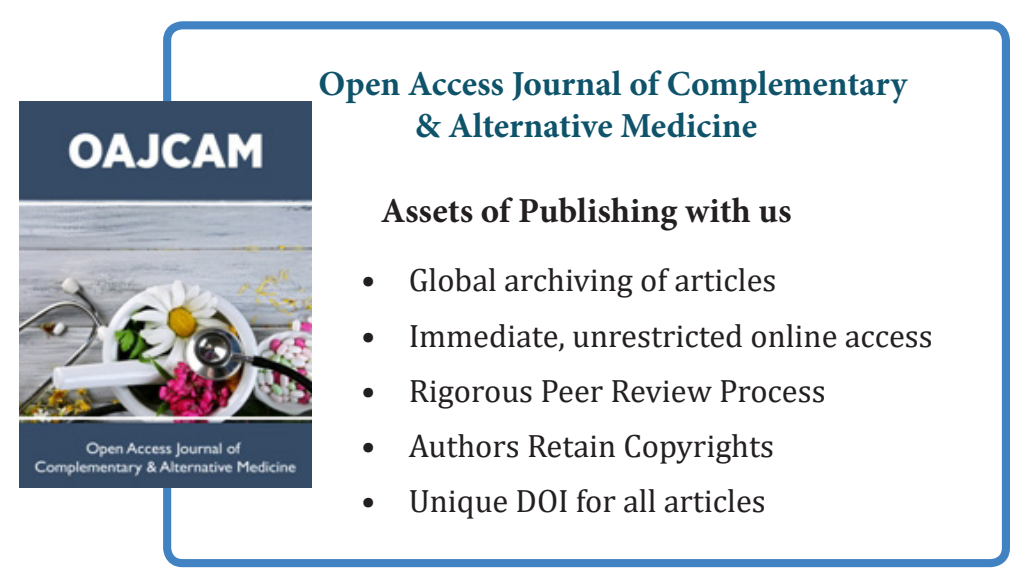

